\title{
Jean Tirole y la nueva identidad de la ciencia económica
}

\author{
Alexander Tobón*
}

Fecha de recepción: 30 de julio de 2020

Fecha de aceptación: 9 de febrero de 2021

Resumen: En La economía del bien común, Jean Tirole marcó un punto de inflexión en la forma de concebir los problemas y métodos utilizados en la economía. Este artículo busca mostrar que esos componentes delimitan una nueva identidad para la ciencia económica, pero sin teoría económica. Para ello, se controvierte la posición de Tirole según la cual la economía debe ser reubicada dentro de las ciencias sociales y humanas. En consecuencia, este artículo contribuye a la reflexión sobre el alcance analítico de la nueva ciencia económica y el significado de lo que hacen los economistas en la actualidad.

Palabras clave: bien común; homo economicus; homo psicologicus; homo socialis; homo juridicus.

Clasificación JEL: B21, B30, C70, C90, D80.

Cómo citar este artículo:

Tobon, A. (2021). Jean Tirole y la nueva identidad de la ciencia económica. Apuntes del Cenes, 40(71). Págs. 17 - 40. https://doi.org/10.19053/01203053.v40.n71.2021.11531

\footnotetext{
* Doctor en Ciencias Económicas, profesor titular del Departamento de Economía, Grupo de Macroeconomía Aplicada, Universidad de Antioquia, Medellín, Colombia. alexander.tobon@udea.edu.co (iD https://orcid.org/0000-0003-1203-9538
} 


\title{
Jean Tirole and Economic Science New Identity
}

\begin{abstract}
In Economics for the Common Good, Jean Tirole sets a turning point in the way of conceiving the problems and methods used in economics. This article attempts to show that these components define a new identity for economic science, but without economic theory. To this end, Tirole's position according to which economics must be relocated within the social and human sciences is refuted. Consequently, this article contributes to reflection on the analytical scope of the new economic science and the meaning of what economists do nowadays.
\end{abstract}

Keywords: common good, homo economicus, homo psychologicus, homo socialis, homo juridicus.. 


\section{INTRODUCCIÓN}

Jean Tirole es uno de los más prominentes contribuyentes a la economía industrial. Su llegada a este campo de la economía se debió a la influencia de Paul Joskow y Dick Schmalensee, sus profesores en el MIT a principios de los años 80. Pero fue la colaboración con Drew Fudenberg y posteriormente con Jean-Jaques Laffont lo que proyectó su intensa trayectoria académica, la cual se evidencia por la considerable cantidad de artículos y libros publicados. Esta trayectoria le mereció muchos reconocimientos, especialmente el Premio Nobel de Ciencias Económicas en 2014. Su interés por las otras ciencias sociales y humanas siempre estuvo vinculado al uso de la teoría de juegos, el lenguaje por excelencia de la teoría de la organización industrial, desde su tesis doctoral bajo la asesoría de Eric Maskin, profesor del MIT e igualmente laureado por el Premio Nobel en 2007. Sin embargo, fue decisiva su colaboración con Ronald Bénabou en el ámbito de la psicología social, desde los inicios de los años 2000.
Publicada por primera vez en 2016, la obra La economía del bien común de Jean Tirole (2017) es uno de los best sellers mundiales. Con seis premios $\mathrm{y}$ trece traducciones a otros idiomas queda clara su significativa aceptación por parte del público académico. En esta obra, su autor asume la responsabilidad que considera debe ser la de todos los economistas: comunicar al público en general los resultados de su investigación y sus opiniones sobre los principales debates económicos. La economía es entonces definida como una cultura que proviene de una comprensión pragmática del mundo, razón por la cual el economista es un intelectual público. En esta perspectiva, Tirole entiende su obra como una herramienta para cuestionar la economía moderna tanto desde un punto de vista teórico, como desde un punto de vista empírico. En efecto, esa fue siempre su pretensión: la teoría pura debería ser probada para que sea aplicada en la toma de decisiones privadas y públicas. Este es el rasgo más notable de la trayectoria académica de Tirole. 
Si bien Tirole autoriza la lectura de los capítulos en el orden que se desee (2017, p. 25), la forma más seductora es considerar las dos primeras partes ("Economía y sociedad" y "La profesión de investigador en economía") como una sola unidad de reflexión, leyendo sus capítulos en el siguiente orden: 4, 2, 1, 5 y 3. Esta unidad de reflexión concentra los componentes básicos de un punto de inflexión en la forma de ver los problemas y los métodos utilizados por la economía, cuyo propósito es la reubicación de la economía dentro de las ciencias sociales y humanas. Por alguna razón desconocida, Tirole considera que la economía es una disciplina separada del resto de aquellas que componen esas ciencias.

El objetivo de este artículo es mostrar que esos componentes delimitan una nueva identidad para la ciencia económica, pero sin teoría económica. En esta nueva identidad, la economía se funda en diversos análisis desarticulados sobre las decisiones humanas en general, con propósitos exclusivamente normativos, especialmente la búsqueda de un bien común.

Para lograr este objetivo, el documento se divide en tres partes. Primero, se presentan los dos antecedentes teóricos necesarios para identificar la fuente de las insatisfacciones por parte de Tirole. En segundo lugar, se expone lo que Tirole llama "el marco teórico unificador" (2017). En tercer lugar, se indican los componentes de la nueva identidad de la ciencia económica. Esos componentes son el concepto de bien común, el cuestionamiento de la ficción neoclásica de homo economicus y el carácter multidimensional del individuo. Al final el lector encontrará las conclusiones de esta reflexión crítica.

\section{ANTECEDENTES TEÓRICOS}

Gran parte de la economía se ha concentrado en el estudio de la coordinación de las decisiones económicas. Por esta vía de investigación, la microeconómica moderna dispone de dos grandes sustentos conceptuales radicalmente distintos: por un lado, la teoría neoclásica del equilibrio general $\mathrm{y}$, por otro, la teoría de juegos. Las dos teorías han dado como resultado, respectivamente, conceptos clave como el equilibrio walrasiano y el equilibrio de Nash. Ambas teorías están explícitamente presentes en Tirole, pero ellas se aprecian de manera muy diferente.

\section{La teoría neoclásica del equilibrio ge- neral walrasiano}

La teoría del equilibrio general constituye el marco analítico de referencia de todo el pensamiento neoclásico. Siguiendo el método positivo, esta teoría proporciona una respuesta sofisticada al paradigma fundador de la economía como disciplina autónoma, propuesto por Adam Smith. Se trata de saber cómo una multitud de individuos, guiados únicamente por sus propios intereses (egoísmo), logran conformar una socie- 
dad económica coherente o no caótica. Según la teoría del equilibrio general, las decisiones individuales pueden coordinarse mediante un sistema de precios de equilibrio, sin la intervención de un Estado o de otra institución exógena a los propios individuos (Hahn, 1981). El fundamento filosófico de esta teoría es el individualismo metodológico, es decir, la idea según la cual la explicación de la sociedad económica tiene como punto de partida el estudio del individuo, definido por una única racionalidad: maximizar sus intereses privados a partir de elecciones alternativas respecto a un conjunto de bienes. Esta es la famosa ficción del homo economicus.

La teoría del equilibrio general describe una economía abstracta compuesta por individuos (consumidores y empresas), a partir de la cual es posible diseñar un sistema de ecuaciones respecto a los bienes y cuya solución es el vector de precios relativos del equilibrio. Este vector de precios es el que iguala la oferta y la demanda en cada mercado, permitiendo que se maximicen los objetivos de los individuos. El rasgo más sustancial de esta teoría es que no existen fricciones o fallos de mercado, lo que se refiere esencialmente a la existencia de dos hipótesis: la competencia perfecta y un sistema completo de mercados. Esta última hipótesis implica que la información de la cual disponen los individuos es simétrica, perfecta y completa, que no hay externalidades de ningún tipo, no hay costos de transacción, ni bienes públicos. Extrañamente, esta parte de la teoría no describe ningún proceso de ajuste de las decisiones individuales, con el fin de explicar cómo se lograría el vector de precios de equilibrio general.

Aunque los economistas neoclásicos reconocieron muchas de las dificultades de esta teoría, todos concuerdan en un resultado altamente significativo: la equivalencia entre las asignaciones de bienes obtenidas por medio de los precios de equilibrio general y aquellas que verifican el criterio de la eficiencia $u$ óptimo de Pareto, lo cual ha conducido a la formulación de los dos teoremas del bienestar. Este resultado permitió que la teoría neoclásica se desarrollara en diferentes perspectivas y se consolidara como la teoría económica dominante, tanto en la formación de los economistas como en la investigación.

Tirole se refiere a la teoría neoclásica del equilibrio general walrasiano mediante la expresión "modelo competitivo" (2017, p. 117). Descrito de forma extremadamente resumida en un recuadro, él observa dos deficiencias:

Esta fue una etapa importante del desarrollo de la teoría económica, pero presenta dos defectos vinculados entre sí. Por una parte, sus implicaciones en la política económica no eran evidentes: la ausencia de fricción (mercados competitivos, información simétrica, racionalidad, etcétera) hace que los merca-

1 Para una presentación sintética de la teoría del equilibrio general, ver Pignol (2017). 
dos sean eficaces. [...]. Por otra parte, ese modelo no describe prácticamente ninguna de las situaciones que abordo en este libro. (Tirole, 2017, p.117)

En estos términos, el modelo competitivo sería inadecuado porque la ausencia de fallos de mercado limita su aplicación en la toma de decisiones económicas de la vida real, que es justamente la pretensión de toda teoría. Es por esta vía que Tirole interroga el carácter científico de la economía y remite a Rodrik (2016). Cree que el método deductivo sobre el cual se ha construido la economía le otorga un "funcionamiento científico", pero no es una ciencia exacta, porque su capacidad de predecir los fenómenos económicos es bastante restringida. La razón es que la economía enfrenta dos obstáculos (2017, pp. 105-106). Primero, la dificultad de disponer de datos, y segundo, no hay objetividad. En efecto, dado que los economistas son individuos, ellos mismos son parte del objeto de estudio en su propia disciplina. Este obstáculo es infranqueable no solo para la economía, sino también para las ciencias sociales y humanas.

\section{Teoría de juegos en el enfoque individual}

La teoría de juegos es hoy en día una de las herramientas más usadas en economía gracias a dos características: su capacidad para dotar de una cierta racionalidad a los jugadores y su capa- cidad de explicar las interacciones entre ellos. Es así como los economistas ven una alternativa a la teoría económica del equilibrio general.

Un juego es la formalización matemática del conjunto de elecciones que pueden realizar los jugadores, basándose en reglas previamente establecidas. En un juego es necesario especificar al menos tres tipos de reglas: (i) La forma de organización del juego. (ii) Las diferentes soluciones posibles del juego asociadas con las ganancias resultantes para cada jugador (positivas en forma de recompensas o negativas en forma de pérdidas o castigos). (iii) Todas las estrategias de actuación disponibles en desarrollo del juego. El modelista (el economista o experimentador) está obligado a explicitar todas las hipótesis que se deducen de estas tres reglas, a diferencia de la teoría del equilibrio general donde hay muchas hipótesis implícitas.

En lo que respecta a la regla (i), se pueden distinguir dos tipos de juegos clásicos: por un lado, los juegos no cooperativos, donde los jugadores considerados individualmente compiten en función de sus propios intereses y, por otro lado, juegos cooperativos, donde los jugadores conforman coaliciones que compiten en función de los intereses de la coalición. Dado que el comportamiento egoísta siempre está presente, estos dos tipos de juegos clásicos constituyen un enfoque 
llamado aquí "individual" 2 . Recordemos ciertos atributos esenciales que facilitan su comparación con la teoría económica del equilibrio general.

En primer lugar, en los juegos no cooperativos se valora la racionalidad individual, las soluciones son generalmente subóptimas y prevalece el concepto de equilibrio de Nash. Este equilibrio designa cualquier combinación de estrategias (de un par de jugadores), de modo que ningún jugador se arrepienta de su elección después de haber verificado la elección del otro, por lo que ningún jugador está interesado en cambiar unilateralmente la estrategia adoptada. En este sentido, Tirole recuerda que el equilibrio de Nash, solución ilustrada por el emblemático "dilema del prisionero", ofrece una respuesta interesante para los economistas (2017, pp. 125-127). Dado que este juego adopta estrategias estrictamente dominantes, ninguno de los acusados puede decidir anticipando lo que hará el otro. Entonces hay un tratamiento del problema de la coordinación de las decisiones individuales, en términos cercanos al paradigma de Smith (citado por Tirole, 2017)3.

En segundo lugar, en los juegos cooperativos se valoran las coaliciones entre jugadores y las soluciones resultan ser a menudo óptimas de Pareto. Al diseñar estos juegos, se deben especificar los acuerdos que establecen los grupos de jugadores. Sin embargo, no es necesario especificar cómo se forman y se mantienen las coaliciones, ni el propósito de compartir las ganancias. Resulta entonces inevitable pensar que este tipo de juegos evoca la existencia de algún tipo de institución (como por ejemplo la confianza), la cual facilitaría una coordinación de los intereses individuales, previa a la conformación de las coaliciones.

Fue en el contexto de los juegos cooperativos que se estableció un resultado importante para la teoría económica: el "principio de equivalencia". Según este principio, la distribución de bienes por medio de un sistema de precios de equilibrio en competencia perfecta es equivalente a la distribución de bienes por medio de "pequeñas negociaciones" sin precios, modelada por la teoría de juegos (Aumann, 2008, pp. 546-548). Este resultado fue obtenido por Shapley y Shubik utilizando el concepto de núcleo propuesto por Edgeworth ${ }^{4}$ (citados por Monsalve, 2018).

Así mismo, resulta interesante saber que antes de que se estableciera este principio de equivalencia, existía otro vínculo entre la teoría del equilibrio general y

2 Este es el sustento del "programa de Nash", el cual establece que, bajo ciertas condiciones, los juegos cooperativos pueden reducirse a juegos no cooperativos.

3 Para el punto de vista de Tirole (2017) sobre el egoísmo en Smith, vea las páginas 61-64.

4 Una síntesis sobre los vínculos entre la teoría neoclásica del equilibrio general y la teoría de juegos se encuentra en Monsalve (2018, pp. 415-434). Así mimo, una breve presentación sobre la contribución de Lloyd Shapley y Martin Shubik puede leerse en Monsalve (2018, pp. 348-354). 
la teoría de juegos. En efecto, la demostración matemática de la existencia del equilibrio de Walras y la del equilibrio de Nash recurren a la aplicación del teorema del punto fijo de Kakutanis. Sin embargo, no hay nada conceptual que vincule ambas teorías, ni siquiera el significado de la palabra "equilibrio". Según Guerrien (1995, p. 35), un equilibrio económico traduce una situación de armonía de las decisiones en la que ninguna fuerza interna o externa (en un sentido físico) la modifica. Sin embargo, en la teoría de juegos no es posible aplicar el concepto de fuerza (Sorin, 1999).

\section{MARCO TEÓRICO UNIFICADOR}

Sin volver sobre los defectos de la teoría del equilibrio general walrasiano, Tirole afirma que la microeconomía moderna se basa en la teoría de juegos -que representa y predice las estrategias de unos actores provistos de objetivos propios e independientes $-\mathrm{y}$ en la teoría de la información- que da cuenta del uso estratégico de informaciones privilegiadas por esos mismos actores. (Tirole, 2017, p. 123)

Estas dos teorías conforman el marco teórico unificador, con el cual la economía encuentra su lugar en las ciencias sociales y humanas.
El marco teórico unificador es una metodología aplicada en diferentes problemas relacionados con el estudio de las decisiones humanas, caracterizada por tener una vocación esencialmente normativa. Tirole insiste en la necesidad de comprobar los postulados teóricos para poder "cambiar el mundo" (2017, p. 101). Ahora, esta comprobación pasa por el diseño de juegos que se aplican bajo la forma de experimentos en laboratorio, donde las decisiones de los jugadores están totalmente controladas. La vocación normativa de la economía deseada por Tirole es de naturaleza distinta a la de Friedman (2017, p. 113)6, pues no cree que la comprobación de los postulados teóricos pase necesariamente por el uso de la econometría (2017, p. 132).

En esta perspectiva, el marco teórico unificador no conduce a la formulación de una teoría general de la sociedad económica (o una teoría globalizadora, 2017, p. 118). Así es como Tirole promueve el abandono de la vocación positiva que ha caracterizado la construcción y el desarrollo de la teoría económica. Veamos ahora cuál es el contenido de las teorías que componen este marco unificador.

\footnotetext{
5 Según Aumann (2008, p. 553), la primera prueba de la existencia de un equilibrio competitivo realizado por Kenneth Arrow y Gérard Debreu en 1954, utiliza la demostración de la existencia de un equilibrio estratégico en un juego generalizado (o restringido), propuesto por Debreu (1952).

6 En esta traducción de la obra de Tirole se omitió por error el nombre de Milton Friedman.
} 


\section{Teoría neoclásica de la información}

La teoría neoclásica de la información o la teoría de los contratos o teoría del agente-principal-agente (2017, p. 129), es el hilo conductor de todo el trabajo de Tirole (p. 24). Esta teoría se construyó a partir de la aplicación de un método propuesto por Arrow (1969), el cual consiste en determinar los precios en un modelo de equilibrio general en presencia de fallos de mercado. Tirole entiende este método como un proceso de introducción de fricciones respecto al "antiguo" modelo de equilibrio general (2017, p. 118). Este proceso de refinamiento en las hipótesis se caracteriza por el hecho de que las restricciones que enfrentan los individuos son ahora más diversas y complejas.

En la teoría del equilibrio general, la hipótesis de un sistema de mercado completo implica la existencia de información simétrica entre individuos. Esto significa que los precios reflejan correctamente la calidad de los bienes para los cuales se determinan los precios. La teoría de la información también tiene como objetivo determinar los precios, pero suponiendo que los individuos disponen de información asimétrica, es decir, no todos saben lo mismo sobre la calidad de los bienes. El ejemplo tradicional de esta idea es el mercado de vehículos usados, planteado por Akerlof
(1970). Uno de los más importantes representantes de la teoría de la información en esta perspectiva es Laffont (1980)7. Su trabajo presenta un modelo neoclásico de equilibrio general, bajo la hipótesis de información asimétrica. En respuesta a la incertidumbre sobre la calidad de los bienes, los individuos deben anticipar, razón por la cual aparecen los comportamientos de riesgo moral y selección adversa. En consecuencia, Laffont muestra que los precios de equilibrio general pierden su papel como un mecanismo eficiente de asignación de recursos. Luego, la eficiencia puede ser eventualmente restablecida mediante la introducción de un medio privado: la creación de un mercado de seguros, centrado en el establecimiento de un contrato que internalice las asimetrías de información8.

Para Tirole, el contrato es el punto de partida del estudio de las decisiones humanas en general, ya que implica la existencia de diferentes tipos de instituciones. Por ejemplo, el tribunal de justicia en presencia de contratos de seguros analizado por Laffont (1980, p. 95). Así es como Tirole considera fundamental el estudio de los comportamientos que vinculan a los individuos con las instituciones. "El tema de la información está en todas partes, en el núcleo mismo de la elaboración de nuestras instituciones y de nuestras decisiones en el ámbito de

7 Tirole también cita a otros economistas neoclásicos (2017, p. 129).

8 La existencia de externalidades es otro fallo de mercado destacado por Tirole. A su juicio, la política económica de un país se basa en el estudio de este fallo, ya que allí es posible observar "la diferencia entre la racionalidad individual y la racionalidad colectiva: lo que es bueno para un actor económico puede no serlo para la sociedad en su conjunto" (Tirole, 2017, p.137). 
la política económica. En el núcleo de la economía del bien común" (Tirole, 2017, p. 25). Tirole está convencido de que, en lugar de considerar los precios como el mecanismo perfecto para la conciliación mutua de los intereses individuales, lo importante es comprender las instituciones como el medio de conciliación del interés individual con el interés colectivo en un mundo con fallos de mercado.

\section{La teoría de juegos en el enfoque colectivo}

La teoría de juegos es la herramienta matemática que sirve de lenguaje a la teoría de la información centrada en las instituciones. Desde luego, la teoría de juegos en el enfoque individual debe ser adaptada para incorporar las decisiones colectivas, es decir, juegos en los cuales se estudie la reacción consciente de un jugador frente a un grupo de otros jugadores. Se trata de abandonar el postulado del egoísmo como la única racionalidad humana contenida en la teoría de juegos clásica en el enfoque individual (2017, p. 127).

Para ilustrar el sentido de los juegos en el enfoque colectivo, Tirole vuelve al juego del "dilema del prisionero". Como se dijo, los dos acusados colectivamente prefieren cierta coordinación antes del juego, pero esto es imposible porque una de las reglas son las estrategias dominantes, es decir que ninguno decide anticipando la decisión del otro. Por lo tanto, la cooperación solo es posible si se elimina esa regla.

El origen de los juegos en el enfoque colectivo se puede encontrar en dos fuentes bien conocidas: por un lado, las obras de Shubik $(1982,1984)$ en el contexto de los juegos cooperativos y, por otro lado, los trabajos de Harsanyi (1967-68) en el contexto de los juegos no cooperativos9. Las obras de Shubik se consideran el punto de partida para muchas aplicaciones de la teoría de juegos en las ciencias sociales y humanas. Según Tirole,

una de las características de las ciencias sociales y humanas es la importancia de las predicciones $y$, especialmente, la comprensión de cómo el [entorno] del agente va a evolucionar y a reaccionar ante sus decisiones: para jugar, un actor debe prever lo que harán los otros actores. (Tirole, 2017, p. 123)

En este sentido, anticipar se define como la capacidad de un jugador de ponerse en el lugar de otros jugadores (p. 128).

El aspecto esencial aquí es que son las anticipaciones las que hacen posible pasar del análisis exclusivamente individual (egoísta tradicional de Smith), al análisis simultáneo del comportamiento individual y colectivo (2017, p. 123). Por lo tanto, cualquier juego que incorpore la capacidad de anticipar como una regla que orienta la toma de decisiones

9 Una síntesis del proceso de desarrollo de la teoría de juegos se puede encontrar en Aumann (2008). 
debería ser capaz de mostrar que la cooperación emerge como un comportamiento espontáneo. Según Tirole, en algunos juegos de laboratorio se ha encontrado que entre el $15 \%$ y el $20 \%$ de los jugadores optan por la cooperación (2017, p. 127), mientras que en el mundo real los individuos suelen ser menos generosos que en el laboratorio (2017, p. 527) ${ }^{10}$.

La teoría de juegos dinámicos con información incompleta aparece como el escenario idóneo, ya que vincula las anticipaciones con los comportamientos individual y colectivo (2017, p. 128)11. Se trata de juegos en los cuales los individuos toman sus decisiones de manera secuencial, primero decide un jugador, luego otro y así sucesivamente. A la excepción del primer jugador, los demás jugadores (la colectividad) deberán anticipar las decisiones de los jugadores que los preceden antes de poder decidir ellos mismos. En presencia de la regla de información incompleta, los jugadores conforman un sistema de creencias, a las cuales se les aplica la teoría de las probabilidades, especialmente el teorema de Bayes. Este procedimiento analítico se remonta al trabajo de Harsanyi (1967-68).

En conclusión, el marco teórico unificador permite concebir la racionalidad del individuo en la siguiente sucesión: el individuo debe tomar decisiones de acuerdo con la información disponible. Si esta última es imperfecta, asimétrica o incompleta, entonces el individuo es víctima de incertidumbre, razón la cual debe anticipar. Tomados en su conjunto, los individuos se cubren de los errores en sus anticipaciones a través de dos medios: establecer contratos que buscan mantener los intereses individuales o cooperar alrededor de un interés común o colectivo. En ambos casos se hacen evidentes las instituciones, por ejemplo, la confianza y la generosidad. Si los individuos son puestos en un juego dinámico con información incompleta, se muestra que la sucesión de decisiones permite una convergencia de los intereses de todos los jugadores, es decir, se concilia simultáneamente el interés individual y el interés colectivo. Es así como se dispone de una metodología para el diseño de las políticas económicas y sociales.

\section{LA NUEVA IDENTIDAD DE LA CIENCIA ECONÓMICA}

El marco teórico unificador se presenta como una forma específica de definir la economía únicamente en torno al estudio de la coordinación de las decisiones humanas. Bajo este formato, la economía cumple el requisito para ser reubicada dentro de las ciencias sociales y humanasi2, en las cuales están la psi-

\footnotetext{
$10 \mathrm{~N} .{ }^{\circ} 36$.

11 Al respecto, ver Fundenberg y Tirole (1991, parte IV).

12 Tirole (2017) no distingue entre ciencias sociales y ciencias humanas.
} 
cología, la sociología, la antropología, el derecho, la historia, la filosofía y la ciencia política (Tirole, 2017, pp. 21, 137).

Este retorno metodológico es posible porque tanto la economía como estas disciplinas se refieren a "las mismas personas, los mismos grupos y las mismas organizaciones" (2017, pp. 137138). A juicio de Tirole,

es indispensable que los diversos campos disciplinarios en ciencias humanas y sociales se abran a los otros y se alimenten entre sí. El economista tiene mucho que aprender de las otras disciplinas y, a la inversa, sus trabajos pueden abrir nuevas perspectivas de investigación sobre los comportamientos individuales y los fenómenos sociales. (2017, p. 138)

En parte, este proyecto de reubicación de la economía en las ciencias sociales y humanas fue inaugurado, entre muchos otros, por James Buchacan, Ronald Coase, Daniel Kahneman, Robert Shiller, Richard Thaler y Elionor Ostrom, ganadores del Premio Nobel de Economía.

Dos argumentos justifican el fin de la autonomía de la economía como disciplina: el compromiso eludido de los economistas con la búsqueda del bien común y el fracaso de la teoría neoclásica basada en la ficción del homo economicus. Ambos argumentos son útiles para comprender el carácter multidimensional del individuo que es absolutamente necesario en cualquier estudio interdisciplinario.

\section{El bien común}

Extrañamente, Tirole se refiere a la noción de bien común solo en el prólogo de su obra. La discusión gira en torno a dos preguntas: por un lado, la definición del bien común en las ciencias sociales y humanas $y$, por otro lado, la búsqueda del bien común por medio de la economía. La siguiente cita presenta los elementos más relevantes sobre estas dos proposiciones:

Ya seamos políticos, empresarios, asalariados, parados, trabajadores independientes, altos funcionarios, agricultores, investigadores, sea cual sea el lugar que ocupemos en la sociedad, todos reaccionamos a los incentivos a los que nos enfrentamos. Estos incentivos -materiales o sociales-, unidos a nuestras preferencias, definen nuestro comportamiento. Un comportamiento que puede ir en contra del interés colectivo. Esa es la razón por la que la búsqueda del bien común pasa en gran medida por la creación de instituciones cuyo objetivo sea conciliar lo más posible el interés individual y el interés general. En este sentido, la economía de mercado no es en absoluto una finalidad. Es, como mucho, un instrumento, $\mathrm{y}$ un instrumento muy imperfecto, si se tiene en cuenta la discrepancia que puede haber entre el interés privados de los individuos, de los grupos sociales o las naciones y el interés general. (Tirole, 2017, p. 15) 
Primero, el bien común se define como el "bienestar colectivo" (2017, p. 16) y es la propia sociedad quien lo decide. Para comprender esta definición, Tirole retoma un instrumento de razonamiento propuesto por Rawls (citado en Tirole, p. 14), llamado colocarse "detrás del velo de la ignorancia". El velo de la ignorancia es un mecanismo que permite establecer un criterio de justicia social: una sociedad justa es aquella en la cual los individuos que la componen ignoran el grupo social al que pertenecen, de modo que la acción emprendida por un individuo para promover su posición social, también promueve la posición de los demás. Tal acción es, por lo tanto, justa socialmente. Así, "detrás del velo de la ignorancia" convergen el interés individual y el interés colectivo.

Sin embargo, Tirole destaca el otro lado de este instrumento de razonamiento. Cuando nos colocamos "frente al velo de la ignorancia", los individuos se reconocen en el grupo social al que pertenecen. Por lo tanto, ellos reaccionan a los incentivos (materiales o sociales) a los que se enfrentan constantemente. $\mathrm{Si}$ las reacciones de un individuo van en contra del interés colectivo, es decir, en contra del bien común, entonces se observa injusticia social. Según Tirole, es allí donde deben crearse instituciones para conciliar los intereses individuales y colectivos, cuyo resultado sería el mantenimiento del bien común. Esto evitaría, por ejemplo, la famosa "tragedia de los bienes comunes" de Hardin (citado en Tirole, p. 128)13.

Segundo, con respecto a la búsqueda del bien común, Tirole considera que "la economía está al servicio del bien común" (2017, p. 17). Subraya claramente dos roles: por un lado, la economía debe dar lugar a debates que permitan distinguir los objetivos establecidos en el bien común, de los instrumentos que permiten su logro. Estos instrumentos son, por ejemplo, instituciones como el mercado, una ley o una política económica. Por otra parte, la economía, al considerar el bien común como un criterio fundamental, desarrolla los instrumentos para contribuir a él (2017, p. 17).

\section{El cuestionamiento del homo economicus}

Tirole está convencido de que la ficción del homo economicus, sobre la que se construyó todo el pensamiento económico neoclásico, es insostenible. La razón es que muestra una racionalidad humana excesivamente simplista y no permite dar cuenta de los innumerables errores cognitivos cometidos por los individuos en la toma cotidiana de sus decisiones. Este cuestionamiento es sorprendente, pues la teoría de la información está construida sobre esa ficción, es decir, un individuo que 
busca maximizar un objetivo individual a partir de un conjunto de alternativas. Sin embargo, el punto esencial para Tirole es que, contrariamente al homo economicus, el ser humano no busca siempre su interés individual material. Al respecto afirma:

Evidentemente, no actuamos siempre en pro de nuestro interés material, lo que, por ejemplo, maximizaría nuestra cuenta bancaria o, en un sentido más general, los bienes y servicios de que disponemos. Hacemos donaciones a organizaciones caritativas, ayudamos a desconocidos que sabemos que no volveremos a ver y de los que no esperamos ninguna reciprocidad. Añadir un componente de empatía a la descripción del objetivo de los actores económicos no entra en contradicción con la teoría económica clásica, pues basta con redefinir el interés bien entendido; si internalizo una parte del bienestar de usted, pasará a ser, de hecho, el mío. (Tirole, 2017, p. 142)

Según Tirole (2017), Smith formó una idea del mercado en torno al egoísmo como un sentimiento o pasión, es decir, sobre la base de la filosofía moral. Los argumentos de Tirole conducen a una pregunta: jel estudio del mercado basado en el egoísmo smithiano debilita la idea de un vínculo social? Refiriéndose a Seabright (2012), Tirole considera que, si el mercado es un dispositivo resultante de la divergencia de intereses, no tiene por qué implicar el rechazo de un vínculo social. La sociedad de mercado, lejos de depender únicamente del egoísmo de los individuos, también exige una gran capacidad de confianza, y nada es más corrosivo para la confianza que el egoísmo puro (2017, p. 63). Para Tirole, "el mercado es un lugar de competición y de colaboración, y el equilibrio entre ambos es siempre delicado" (p. 63).

Ahora, aunque Tirole cuestiona la ficción del homo economicus, no rechaza el individualismo metodológico, el cual considera "es perfectamente compatible (incluso quizá indispensable) con la comprensión y el análisis preciso de los fenómenos de grupo. Los actores económicos reaccionan a sus incentivos y algunos de esos incentivos proceden de los grupos sociales a los que pertenecen" (2017, p. 102). Los incentivos que se encuentran en el centro de la definición del bien común están vinculados a esta interpretación del individualismo metodológico ${ }^{14}$.

La propuesta de Tirole se dibuja entonces de la siguiente manera: las ciencias sociales y humanas (incluida la economía) deben estar dotadas de una metodología que sea tanto individualista como holística, es decir, una metodología que considere el comportamiento individual y comportamiento colectivo como complementarios (2017, p. 156). Para hacer esto, el individuo debe definirse tanto en su dimensión individual como

14 Tirole es consciente de que muchos investigadores en ciencias sociales y humanas no se adhieren al individualismo metodológico (2017, p. 138). Para una crítica desde el punto de vista de la sociología, ver Durand y Weill (1989). 
en su dimensión social (p. 17). En otras palabras, los comportamientos humanos que interesan son aquellos que concilian simultáneamente el interés privado y el interés colectivo. Si se demuestra que esta conciliación de intereses pasa por la existencia de instituciones, la realización del bien común es posible.

\section{El carácter multidimensional del individuo}

Las decisiones humanas se toman en función de ciertos comportamientos, los cuales están determinados por un entorno multidimensional complejo. Según Tirole (2017), el comportamiento individual se inscribe al menos en cuatro ámbitos: económico, psicológico, social y jurídico. En lo referente al ámbito económico, Tirole usa la expresión homo incitatus para identificar los comportamientos individuales frente a ciertos incentivos no siempre monetarios. A partir de ahí, propone agrupar los comportamientos en las tres expresiones siguientes: homo psicologicus, homo socialis y homo juridicus. Es el homo psicologicus el que domina ampliamente la exposición de Tirole.

La aplicación del marco teórico unificador (con frecuencia implícito) muestra que más allá de determinar una situación de armonía de las decisiones llamada "equilibrio", lo que interesa ahora son los dilemas que enfrentan los individuos cuando deciden. El problema tradicional de la economía -como disciplina autó- noma- de estudiar la coordinación de individuos por medio del intercambio de bienes en el mercado, es solo un caso particular dentro del conjunto de todas las decisiones humanas.

\section{“Homo incitatus"}

En el ámbito económico, homo incitatus se define por un conjunto de argumentos destinados a mostrar que los incentivos están en la base del comportamiento humano, pero no se trata siempre de incentivos monetarios o materiales. De esta manera, homo incitatus reemplaza a homo economicus. Según Tirole, existen casos en los cuales los incentivos monetarios de homo economicus no conducen al logro del objetivo buscado, lo que muestra que el mercado $\mathrm{y}$, por lo tanto, los precios, no siempre son el mejor mecanismo para coordinar las decisiones económicas.

Este punto de vista se encuentra en Bénabou y Tirole (2003), bajo la tesis de que la motivación extrínseca puede desplazar la motivación intrínseca, haciendo que haya incentivos contraproducentes. El ejemplo de la donación de sangre resalta este efecto (2003, pp.158162). Cuando se ofrecen recompensas monetarias para los donantes de sangre (un bien de interés colectivo) con la esperanza de aumentar la cantidad de donaciones, se tiene el resultado contrario. El donante teme que la recompensa sea interpretada por el resto de los individuos como un signo de avaricia 
(motivación extrínseca), en lugar de generosidad (motivación intrínseca). Tirole destaca un comportamiento psicológico fundamental en la toma de las decisiones humanas: la preocupación de aparentar, es decir, la imagen que el individuo proyecta hacia los demás (2017, p. 159). Si este comportamiento psicológico no existiera (como en homo economicus), el incentivo monetario conduciría efectivamente a un aumento en la donación de sangre. Para Tirole, muchas decisiones sobre bienes de interés colectivo están dominadas por este comportamiento psicológico.

Los comportamientos que encuentran su explicación en psicología traducen una racionalidad humana compleja, la cual puede estudiarse definiendo un homo psicologicus. Según Tirole, la psicología social explica muchos comportamientos que permiten ir más allá de la explicación basada en el egoísmo smithiano.

\section{"Homo psicologicus"}

En su trabajo, Tirole distingue los comportamientos psicológicos puestos en una perspectiva tanto individual como prosocial. La teoría de juegos y la teoría de la información (el marco teórico unificador) hallan aquí un campo de aplicación inesperado, pero bastante natural (2017, p. 151).

\section{- Desde la perspectiva individual}

Reúne los comportamientos psicológicos que van en contra del interés individual, es decir, la racionalidad del homo economicus. Tirole identifica cuatro comportamientos psicológicos importantes: la procrastinación, los errores en la formación de creencias, la empatía y la automanipulación de creencias 15 . La Tabla 1 presenta la definición de estos comportamientos psicológicos y un ejemplo.

Tabla 1. Comportamientos individuales del homo psicologicus

\begin{tabular}{|c|c|}
\hline $\begin{array}{l}\text { Comportamientos psicológicos que } \\
\text { van en contra del interés individual }\end{array}$ & Ejemplos \\
\hline $\begin{array}{l}\text { Procrastinación. Postergar una acción prioritaria } \\
\text { para atender en el presente una acción más } \\
\text { agradable. Es una elección intertemporal que } \\
\text { muestra una preferencia por el presente o por el } \\
\text { corto plazo. }\end{array}$ & $\begin{array}{l}\text { Quiero dejar de fumar, sin embargo hoy quiero fumar } \\
\text { mi último cigarrillo y dejaré para mañana la dolorosa } \\
\text { tarea de no fumar. }\end{array}$ \\
\hline $\begin{array}{l}\text { Errores en la formación de creencias. Una creencia } \\
\text { es la capacidad mental de un individuo para } \\
\text { calcular una probabilidad (estadística) sobre la } \\
\text { ocurrencia de un evento futuro. }\end{array}$ & $\begin{array}{l}\text { Supongamos que se tira al aire una moneda para } \\
\text { que caiga cara o sello. Aunque sabemos que la } \\
\text { probabilidad es siempre del } 50 \% \text {, creemos que } \\
\text { cuando la moneda ha caído sello tres veces seguidas, } \\
\text { la probabilidad de que en el próximo tiro caiga cara } \\
\text { es más del } 50 \% \text {. Sin embargo, la moneda no tiene } \\
\text { memoria. Es la falacia del apostador. }\end{array}$ \\
\hline
\end{tabular}

15 Tirole también menciona el optimismo excesivo, la fuerte aversión a las pérdidas, el papel contraproducente de las emociones y la memoria selectiva (2017, p. 142). 
Empatía. Es la capacidad de un individuo de Las donaciones a organizaciones benéficas: la internalizar de manera interesada el bienestar de felicidad de los pobres contribuye a la felicidad del otro individuo en su propio bienestar. donante.

Automanipulación de creencias. Eliminar, olvidar Dado que me veo inteligente, hermosa y generosa, o reinterpretar información desfavorable para decido buscar un empleo como presentadora de tener una mejor imagen de sí mismo y tomar una televisión. decisión que no se tomaría en otras condiciones (por pereza, miedo o procrastinación).

\section{- Desde la perspectiva prosocial}

Se refiere a los comportamientos individuales prosociales en los cuales el interés material no tiene la prioridad, es decir, el individuo internaliza desinteresadamente el bienestar de los demás (2017, p. 143). Se trata de comportamientos individuales como el altruismo, el cual es motivado por tres factores: la motivación intrínseca, la motivación extrínseca y el deseo de aparentar, es decir, dar una buena imagen de sí, tanto a los otros como a sí mismo (p. 144). La Tabla 2 muestra que, según Tirole, el altruismo es un comportamiento complejo: puede ser interesado, recíproco y frágil.

Tirole destaca otros dos comportamientos psicológicos individuales desde una perspectiva prosocial: la fragilidad de la honestidad debido a la pérdida de la memoria (2017, p. 150) y el castigo por el exceso de altruismo puro (pp. 150-151).

Tabla 2. Comportamientos prosociales del homo psicologicus

\begin{tabular}{|c|c|}
\hline Conductas psicológicas prosociales & Ejemplos \\
\hline $\begin{array}{l}\text { Altruismo vinculado a la imagen de sí mismo. Un } \\
\text { individuo es generoso con la condición de que } \\
\text { todos los demás vean su generosidad, porque es el } \\
\text { prestigio social lo que le interesa. }\end{array}$ & $\begin{array}{l}\text { Hay más donaciones a un museo cuando los } \\
\text { donantes se hacen públicos. }\end{array}$ \\
\hline $\begin{array}{l}\text { Altruismo recíproco o cooperativo. Un individuo es } \\
\text { generoso solo con aquellos que también lo son con } \\
\text { él (según las preferencias sociales). }\end{array}$ & $\begin{array}{l}\text { Las personas que conforman la microsociedad de } \\
\text { los Tsimanes en Bolivia y los Hadzas en Tanzania } \\
\text { (Tirole, 2017, p. 146). }\end{array}$ \\
\hline $\begin{array}{l}\text { Altruismo frágil por excusas. Frente a la elección de } \\
\text { una acción egoísta o altruista, el individuo prefiere } \\
\text { no tener que elegir. }\end{array}$ & $\begin{array}{l}\text { Un individuo que cambia la acera cuando ve a una } \\
\text { persona sin hogar en la distancia que va a pedirle } \\
\text { dinero. Así evita enfrentar la decisión de dar o no } \\
\text { dar dinero. }\end{array}$ \\
\hline $\begin{array}{l}\text { Altruismo frágil debido al contexto. Frente a la } \\
\text { elección de una acción altruista, otra egoísta y otra } \\
\text { muy egoísta, la acción egoísta parece mejor que la } \\
\text { acción muy egoísta. La relativización del egoísmo } \\
\text { debilita el altruismo. }\end{array}$ & $\begin{array}{l}\text { El Estado ofrece a una persona un plan de ahorro } \\
\text { para su jubilación. Le dicen que este plan es } \\
\text { adecuado para "la mayoría de las personas", es } \\
\text { decir, es un plan altruista. La persona lo acepta } \\
\text { incluso si hay un mejor plan basado en sus propias } \\
\text { preferencias de ahorro. Es un fenómeno sugestivo. }\end{array}$ \\
\hline
\end{tabular}




\section{“Homo sociales"}

Se puede observar al individuo en el contexto o entorno social en el que toma sus decisiones, ya que forma parte de grupos sociales y estos afectan su comportamiento16. La Tabla 3 muestra los dos comportamientos de origen social que afectan la toma de decisiones individuales: la confianza pura en los demás y la reputación colectiva.

Tabla 3. Comportamientos de origen social del homo socialis

\begin{tabular}{|c|c|}
\hline Comportamiento social & Ejemplos \\
\hline $\begin{array}{l}\text { Confianza pura en los demás. Un individuo recopila } \\
\text { información imperfecta sobre la fiabilidad y las } \\
\text { preferencias de otro individuo. Como no se codea } \\
\text { con él, no puede evaluar estos dos elementos, pero } \\
\text { aun así le concede un cierto grado de confianza. }\end{array}$ & $\begin{array}{l}\text { Como empresario, yo pago un salario más alto a un } \\
\text { nuevo trabajador esperando un aumento voluntario } \\
\text { de su productividad. }\end{array}$ \\
\hline $\begin{array}{l}\text { Reputación colectiva y estereotipos. Se trata de } \\
\text { la influencia de un grupo que tiene una cierta } \\
\text { reputación (buena y mala) sobre las decisiones } \\
\text { individuales. La reputación colectiva es un bien } \\
\text { público para la comunidad (Tirole, 2017, p. 156). }\end{array}$ & $\begin{array}{l}\text { La mala reputación de los taxistas por hacer } \\
\text { cobros excesivos a los turistas. Para un taxista } \\
\text { en particular, defender la buena reputación de } \\
\text { los taxistas le trae un alto costo privado y un bajo } \\
\text { beneficio privado, pues este último lo comparte con } \\
\text { todos los demás taxistas. Surge el fenómeno del } \\
\text { free riding: ningún taxista tiene interés en defender } \\
\text { la buena reputación del gremio. }\end{array}$ \\
\hline
\end{tabular}

\section{"Homo juridicus"}

La ley es una expresión de los valores sociales o morales de una sociedad, en la medida en que hace explícitos los comportamientos individuales aceptados y prohibidos. La ley es, por lo tanto, una norma social o una institución. Para explicar esta afirmación, Tirole utiliza un ejemplo muy familiar: la ley sobre la pena de muerte. En Europa existe una norma social según la cual ningún delito justifica la pena de muerte. Por el contrario, en los Estados Unidos, donde existe la pena de muerte, se establece otra norma social: existen delitos socialmente inaceptables.
La idea de Tirole es que los comportamientos prosociales individuales establecidos por ley pueden promoverse de manera más efectiva a través de mensajes publicitarios estratégicos al público, sin la necesidad de incentivos (recompensas o castigos) ${ }^{17}$. Por ejemplo, una ley que ordena a los ciudadanos clasificar sus desechos destinados al reciclaje, bajo la amenaza de una multa. Entonces, para promover el reciclaje, en lugar de incurrir en costos asociados con la búsqueda de infractores a la ley, el Estado debe financiar a bajo costo una campaña publicitaria en la que se anuncie públicamente el porcentaje de ciudadanos que reciclan. Los anuncios

16 Sobre la cuestión de la existencia de una racionalidad colectiva, ver Bassett y Claveau (2011)

17 Al respecto, ver Bénabou y Tirole (2012). 
de este tipo buscan promover virtudes colectivas. Nuevamente se trata de volver a la psicología de los individuos. Este mecanismo también funciona para las normas sociales que no son necesariamente leyes. Por ejemplo, una ciudad que quiere promover una reducción en el consumo de alcohol debe invertir en un anuncio que muestre la cantidad de personas que no beben alcohol y que muestre la cantidad de dinero que puede usarse para otras actividades.

\section{El alcance analítico de la nueva identidad}

A partir de los años 80, la ciencia económica sufrió una ruptura metodológica como consecuencia de la famosa crítica de Lucas. Considerada como un método analítico demasiado estrecho, la teoría económica pura se fue desdibujando, a medida que crecía la exigencia de gobiernos y grandes empresas por obtener recomendaciones concretas, orientadas a aprovechar las transformaciones neoliberales de aquellos años.

La nueva identidad de la ciencia económica propuesta por Tirole es un punto de inflexión de esa ruptura. La economía, como disciplina autónoma, entendió por muchos años que su objeto de estudio era la coordinación de las decisiones económicas por medio del mecanismo del mercado. Sin embargo, para Tirole, la economía, reubicada en las ciencias sociales y humanas, debe contribuir al estudio de la coordinación de todas las decisiones humanas. En este contexto, la economía deja de ser una filosofía de la sociedad, para convertirse en una herramienta con normativos. Algunas críticas a los componentes de la nueva ciencia económica son los siguientes:

1. La ficción del homo economicus es cuestionada porque su comportamiento es puramente económico y excesivamente simplista, guiado por incentivos monetarios que a menudo resultan ser contraproducentes. El comportamiento egoísta smithiano que le dio origen, resulta ser insuficiente para dar cuenta de la complejidad que revisten las decisiones humanas. El paradigma de Smith, fundador de la ciencia económica autónoma, es injustamente considerado como demasiado estrecho para ser mantenido.

2. La teoría de juegos en el enfoque colectivo se presenta como la herramienta idónea para el estudio de todas las decisiones humanas individuales, situadas en un contexto en el que responden al comportamiento de los demás (otro individuo, un grupo de individuos o la sociedad en su conjunto). La teoría neoclásica de la información juega un papel subsidiario, al permitir el diseño de reglas de juego relacionadas con las diferentes restricciones de información a las que se enfrentan los jugadores y las instituciones que surgen a su alrededor. Aunque tenga un papel subsidiario, la per- 
manencia de la teoría neoclásica de la información nos hace pensar que su modelo de referencia -la teoría del equilibrio general- no puede considerarse totalmente obsoleto.

3. Tirole espera obtener un resultado significativo: mostrar que existen juegos en los cuales es posible hacer que el individualismo metodológico sea compatible con el holismo, es decir, juegos que muestran cómo el interés individual puede ser simultáneamente compatible con el interés de la sociedad. Este resultado es contrario al paradigma de Smith, pues en este el interés de la sociedad es solo comprensible después de que todos los individuos han logrado sus propios intereses.

4. Las decisiones individuales tienen su origen en diversos comportamientos psicológicos, tales como la procrastinación, la empatía, el altruismo, la confianza, la automanipulación de las creencias, la reputación colectiva y la sugestión. ¿Permiten estos comportamientos configurar una psicología general cuyos conceptos se fundan coherentemente con aquellos de la economía? La eliminación de las fronteras entre las ciencias sociales y humanas debería ser un fin en sí mismo y no un medio.
5. La nueva ciencia económica aparece conformada por una amplia gama de juegos interdisciplinarios, cuyo objetivo es resaltar los dilemas y paradojas que subyacen en la toma de decisiones individuales. No pretende demostrar la existencia de un equilibrio, en el sentido de una situación de compatibilidad de las decisiones que reflejan una situación de armonía inmutable. El problema del intercambio de bienes privados por medio de un sistema de precios aparece como un caso especial entre un vasto conjunto de decisiones humanas. Para la economía convencional no se trata de un problema banal sino, por el contrario, del problema fundamental que debió ser resuelto por las teorías del valor, las cuales fueron las primeras en darle su estatus de ciencia.

6. Dado que los juegos se prueban por medio de experimentos controlados en el laboratorio con individuos reales, la nueva ciencia económica adquiere una vocación esencialmente normativa, orientada a la búsqueda del bien común y, según la visión de Tirole, esto equivale a reforzar su carácter científico porque los experimentos mejoran la predictibilidad de los fenómenos sociales, ya que arrojan datos estadísticos cuyas regularidades terminan por considerarse "leyes" del comportamiento humano. 
7. Los juegos son ejemplos considerados altamente representativos de las decisiones humanas. La identidad de la nueva ciencia económica representa, por lo tanto, la muerte de la teoría económica positiva tradicional: la construcción de una teoría general de la sociedad económica deja de ser una preocupación intelectual. En síntesis, no se trata de una reubicación de la economía en las ciencias sociales y humanas, sino de su abandono.

\section{CONCLUSIONES}

Jean Tirole se inscribe en la lista de los economistas que desde los años 80 consideran la teoría económica positiva como un campo de análisis demasiado estrecho. Desde su punto de vista, la ficción de homo economicus, centro del análisis neoclásico, es inaceptable. En esencia, esa ficción no recoge los atributos que caracterizan la racionalidad de un individuo, conducentes a separar las decisiones propiamente económicas del resto de las decisiones humanas. En consecuencia, el individuo es multidimensional y requiere un estudio interdisciplinario dentro de las ciencias sociales y humanas.

La teoría de juegos facilita una formalización de la racionalidad contenida en las decisiones de individuos multidimensionales que interactúan entre ellos, a la vez que permite probar con personas reales muchas de las paradojas y los dilemas que sí caracterizan las decisiones humanas. La racionalidad depende, en gran medida, de la información disponible por los individuos. Estos experimentos en laboratorios humanos posibilitan la acumulación de datos estadísticos (que se obtienen por encuestas), los cuales serían interpretados como "leyes" del comportamiento humano. La promesa es entonces disponer de un análisis más amplio, más realista y, sobre todo, verdaderamente práctico para la toma de decisiones privadas y públicas.

Ahora, dentro de todos los juegos imaginables, Tirole busca seducir a sus lectores invocando la posible existencia de un superjuego: aquel que formaliza las reglas conducentes al logro del bien común. El economista debe considerar el premio del juego -el bien comúncomo algo ya conocido, mientras que las reglas del juego se refieren a los incentivos creados por instituciones de toda índole. Más allá de poder diseñar y solucionar este superjuego, es claro que la teoría de juegos es una herramienta matemática flexible y no una filosofía social. El proyecto de la reubicación de la economía dentro de las ciencias sociales y humanas implica el abandono de la teoría económica, sobre la cual se construyó exitosamente la economía como disciplina autónoma. Pero aún es temprano para juzgar la bondad de esta nueva metodología. 
AGRADECIMIENTOS Y DECLA- las actividades del convenio de doble RACIÓN DE CONFLICTO DE titulación entre esa institución y la INTERESES Universidad de Antioquia (Colombia).

El presente artículo se realizó en el marco de la estancia del autor como profesor visitante en la Universidad de Le Mans (Francia), entre el 25 de marzo y el 1 de abril de 2019, como parte de El autor es el único responsable del contenido del artículo y declara que no tiene conflicto de intereses. El autor agradece los comentarios de los evaluadores anónimos de la Revista. 


\section{REFERENCIAS}

[1] Arrow, K. (1969). The Organization of Economic Activity: Issues Pertinent to the Choice of Market Versus Nonmarket Allocation. In Collected Papers of Kenneth J. Arrow, vol. 2. General Equilibrium (pp. 500-518). Belknap Press.

[2] Aumann, R. J. (2008) Game Theory. In S. Durlauf \& L. Blume (Eds.), The New Palgrave Dictionary of Economics vol. 3, (2nd ed.), (pp. 529-558). Palgrave Macmillan.

[3] Bassett, D. \& Claveau, F. (2011) The Economic Entomologist: An Interview with Alan Kirman, Erasmus Journal for Philosophy and Economics, 4(2), 42-66. https:// doi.org/10.23941/ejpe.v4i2.80

[4] Bénabou, R. \& Tirole, J. (2003). Intrinsic and Extrinsic Motivation. Review of Economic Studies, 70(3), 489-520. https://doi.org/10.1111/1467-937X.00253

[5] Bénabou, R. \& Tirole, J. (2012) Laws and Norms. IZA Discussion Paper, (6290). https://ssrn.com/abstract=1999318, https://doi.org/10.3386/w17579

[6] Debreu, G. (1952). A Social Equilibrium Existence Theorem. Proceedings of the National Academy of Sciences of the United States, 38(10), 886-93. https://doi. org/10.1073/pnas.38.10.886

[7] Durand, J. P. \& Weill, R. (1989). Sociologie contemporaine. Vigot.

[8] Fudenberg, D. \& Tirole, J. (1991). Game Theory. MIT Press.

[9] Guerrien, B. (1995). La théorie des jeux, (2eme édi.). Economica.

[10] Hahn, Frank (1981) General Equilibrium Theory. In Bell and Kristol (Eds.), Crisis in Economic Theory (pp. 123-138). Basic Books.

[11] Harsanyi, J. (1967-68) Game with Incomplete Information Played by 'Bayesian' Players, Parts I, II, and II. Management Science, 14, 159-502. https://doi.org/10.1287/ mnsc.14.7.486

[12] Laffont, J-J. (1980). Essays in the Economics of Uncertainty. Harvard University Press.

[13] Monsalve, S. (2018). Competencia bajo equilibrio de Nash. Curso fundamental de Microeconomía, vol. 3. Universidad Nacional de Colombia. 
[14] Pignol, C. (2017). La théorie de l'équilibre général. Presses Universitaires du Septentrion. https://doi.org/10.4000/books.septentrion.34888

[15] Rodrik, D. (2016). Las leyes de la economía: aciertos y errores de una ciencia en entredicho. Deusto.

[16] Seabright, P. (2012). La société des inconnus: histoire naturelle de la collectivité humaine. Editions Markus Haller.

[17] Shubik, M. (1982). Game Theory in the Social Sciences, Volume I. MIT Press.

[18] Shubik, M. (1984). Game Theory in the Social Sciences, Volume II. MIT Press.

[19] Sorin, S. (1999). Von Neuman-Morgenstern, Nash et Arrow-Debreu : théories des jeux et équilibre général. Cahiers, d'économie politique, 35, 7-17. https://doi. org/10.3406/cep.1999.1259

[20] Tirole, J. (2017). La economía del bien común. Taurus, Penguin Random House Grupo Editorial. 